\title{
Discoidin domain receptor 2 regulates the adhesion of fibroblasts to 3D collagen matrices
}

\author{
DAEHWAN KIM ${ }^{1}$, EUNAE YOU ${ }^{1}$, NA YOUNG MIN ${ }^{1}$, KWANG-HO LEE $^{1}$, \\ HYOUNG KYU KIM ${ }^{2}$ and SANGMYUNG RHEE ${ }^{1}$

\begin{abstract}
${ }^{1}$ Department of Life Science (BK21 Program), Chung-Ang University, Seoul 156-756; ${ }^{2}$ Department of Medicine and Microbiology, College of Medicine, Chungbuk National University, Cheungju 361-763, Republic of Korea
\end{abstract}

Received December 18, 2012; Accepted February 22, 2013

DOI: $10.3892 /$ ijmm.2013.1320

\begin{abstract}
The collagen matrix constitutes the primary extracellular matrix (ECM) portion of mammalian connective tissues in which the interaction of the cell and the surrounding collagen fibers has a significant impact on cell and tissue physiology, including morphogenesis, development and motility. Discoidin domain receptors (DDR1 and DDR2) have been identified as the receptor tyrosine kinases that are activated upon collagen binding. However, there is a lack of evidence regarding the effect of DDRs on the mechanical interaction between fibroblasts and ECM. In this study, we demonstrated that one of the major phosphotyrosine proteins in human fibroblasts during 3D collagen matrix polymerization is DDR2. Treatment of fibroblasts in 3D collagen matrices with plateletderived growth factor (PDFG) has been shown to increase DDR2 phosphorylation. Silencing of DDR2 with siRNA in fibroblasts significantly reduced the number of dendritic extensions regardless of whether cells were cultured in the collagen or fibronectin 3D matrices. Decreasing dendritic extensions in DDR2-silenced cells has also been shown to decrease the ability of fibroblast entanglement to collagen fibrils in 3D collagen matrices. Finally, we also showed that the silencing of DDR2 decreased the cell migration in 3D nested collagen matrices but had no effect on 3D floating matrix contraction. Collectively, these results suggest that DDR2 functioning is required for the membrane dynamics to control the mechanical attachment of fibroblasts to the 3D collagen matrices in an integrin-independent manner.
\end{abstract}

\section{Introduction}

The interactions between cells and their surrounding extracellular matrices (ECMs) play a crucial role in normal

Correspondence to: Professor Sangmyung Rhee, Department of Life Science (BK21 Program), Chung-Ang University, 84 Heukseok-ro, Seoul 156-756, Republic of Korea

E-mail: sangmyung.rhee@cau.ac.kr

Key words: discoidin domain receptor 2, phosphotyrosine protein, migration, adhesion, dendritic extension, 3D collagen matrix physiological processes such as tissue development, morphogenesis and cellular differentiation (1-3). Abnormal changes in cell and ECM interaction have been shown to cause diverse pathological conditions, including tissue fibrosis and tumor associated stromagenesis $(4,5)$.

ECM consists of various proteins including collagen and proteoglycan. The interaction of ECM proteins and the cell is known to be mediated by integrin and syndecan, a wellknown ECM receptor existing on the plasma membrane (6). The extracellular domain of the receptors binds to diverse ECM proteins, and induces the recruitment of various signaling molecules and cytoskeleton proteins. Ultimately, this physical and mechanical interaction of the cell and the ECM is connected with functional regulation of various cellular dynamics and tissue homeostasis (7). In particular, integrins can act as a mechanosensor, which convert mechanical signals created by the ECM into biochemical signals in the cells. Series of these events influence tissue-specific biological events reflected in the cell-ECM mechanical interaction $(8,9)$.

Fibroblasts cultured in 3D collagen matrices presented distinct morphological features and signaling compared with those cultured on the more commonly used 2D surface. Cells interacting with 2D planar surface formed in a flattened, lamellar shape with massive focal adhesions and actin stress fibers, while cells in the $3 \mathrm{D}$ collagen matrices were dendritic in shape with long, slender extensions which are similar to the in situ appearance of mesenchymal cells and connective tissue fibroblasts (10). Moreover, dendritic extensions of fibroblasts in the $3 \mathrm{D}$ collagen matrices became entangled with matrix fibrils, resulting in an integrin-independent mechanical interaction (11). However, the underlying molecular mechanisms remain poorly understood.

The discoidin domain receptor (DDR) family, which includes the receptor tyrosine kinase, has recently been identified as a non-integrin receptor for collagen (12). The DDR family is composed of two members, DDR1 and DDR2. DDR1 is primarily expressed in the epithelial cells, particularly of the lungs, kidneys and mammary glands, whereas DDR2 is found in cells which are of mesenchymal origin, such as fibroblasts and smooth muscle cells (13). It has been clearly shown that the activation of DDRs is linked to intracellular signaling, resulting in the control of cell proliferation and transcriptional regulation. Upon collagen-mediated receptor activation, DDRs 
become phosphorylated on several tyrosine residues in their cytoplasmic regions. These tyrosine residues provide binding sites for a number of different Src homology-2 (SH2) and phosphotyrosine binding (PTB)-containing proteins such as Nck, ShcA and PI3 kinase (14). Although it has been demonstrated that DDRs also have an ability to regulate cell adhesion and are active in the remodeling of the ECM, DDRs do not seem to be required for integrin activity as a co-receptor for ECM (15). Moreover, the role of DDRs in the mechanical interaction of cells to the ECM components has not been studied.

In the current study, we found that DDR2 is a major phospho-tyrosinated protein in the $3 \mathrm{D}$ collagen matrices. Incubation of DDR2-silenced fibroblasts with 3D collagen matrices demonstrated that fibroblasts attached to collagen fibrils in 3D collagen matrices is dependent on DDR2. Finally, we also showed that the DDR2 silencing influenced the ability of fibroblasts to migrate in 3D environments but did not affect matrix contraction and remodeling, indicating that active DDR2 is required for the mechanical interaction of fibroblasts to $3 \mathrm{D}$ collagen matrices with control of the dendritic extensions, which in turn appeared to be critical for fibroblast migration in $3 \mathrm{D}$ collagen matrices.

\section{Materials and methods}

Materials. Dulbecco's modified Eagle's medium (DMEM) and $0.25 \%$ trypsin/EDTA and oligofectamine solution were purchased from Invitrogen (Gaithersburg, MD, USA). Fetal bovine serum (FBS) was purchased from HyClone (Logan, UT, USA). Platelet-derived growth factor (PDGF) was obtained from Upstate Biotechnology, Inc. (Lake Placid, NY, USA). Alexa Fluor 488 phalloidin, Alexa Fluor 594 phalloidin and propidium iodide (PI) were obtained from Molecular Probes (Eugene, OR, USA). RNase (DNase-free) was purchased from Roche (Indianapolis, IN, USA). Fluoromount-G was obtained from Southern Biotechnology Associates, Inc. (Birmingham, AL, USA). Primary antibodies were: goat anti-human DDR2 (polyclonal) antibody from R\&D Systems (Minneapolis, MN, USA) and Type I rat tail collagen $(10.6 \mathrm{mg} / \mathrm{ml})$ purchased from BD Biosciences (Bedford, MA, USA). All other chemical reagents were purchased from Sigma (St. Louis, MO, USA) unless otherwise specified.

Cell culture and nested collagen matrices. Early passage of human foreskin fibroblast BR5 cells were cultured in DMEM supplemented with $10 \%$ FBS. Cell culture and experimental incubations were carried out at $37^{\circ} \mathrm{C}$ in a $5 \% \mathrm{CO}_{2}$ incubator.

For experiments with the collagen matrices, cells in neutralized solutions of $1 \mathrm{mg} / \mathrm{ml}$ of collagen were placed in 24-well culture plates or seeded on top of collagen matrices following polymerization ( $2 \times 10^{4}$ cells/matrix). Growth factors and inhibitors were added as described in the figure legend.

To measure cell migration using nested collagen matrices, floating matrices were precontracted for $4 \mathrm{~h}$ in DMEM/10\% FBS, after which the cell-containing contracted matrices (dermal equivalents) were re-embedded in $200 \mu$ l cell-free outer collagen matrices and then incubated for an additional $24 \mathrm{~h}$ in DMEM/BSA + $50 \mathrm{ng} / \mathrm{ml}$ PDGF. At the end of the incubations, samples were fixed and stained with Alexa Fluor-conjugated phalloidin to visualize actin and PI to detect cell nuclei.
Immunoprecipitation. To identify the collagen-induced phosphorylated proteins in tyrosine residues, collagen matrices containing BR5 fibroblasts were polymerized for $1 \mathrm{~h}$ after which the matrices were further incubated in DMEM containing $50 \mathrm{ng} / \mathrm{ml}$ of PDGF for $4 \mathrm{~h}$. Samples were lysed using Dounce homogenizer with a modified RIPA buffer (50 mM HEPES, $\mathrm{pH} 7.5,150 \mathrm{mM} \mathrm{NaCl}, 1.5 \mathrm{mM} \mathrm{MgCl}_{2}, 5 \mathrm{mM}$ EGTA, $10 \%$ glycerol, $1 \%$ Triton X-100, $10 \mu \mathrm{g} / \mathrm{ml}$ aprotinin, $10 \mathrm{mM} \mathrm{NaF}$, $1 \mathrm{mM}$ PMSF and $1 \mathrm{mM}$ sodium orthovanadate). After clearing by centrifugation, cell lysates $(\sim 2 \mathrm{mg})$ were mixed with antityrosine (PY20) DDR2 antibodies overnight at $4^{\circ} \mathrm{C}$ and then with $100 \mu \mathrm{l}$ of $30 \%$ slurry of protein A-sepharose for $2 \mathrm{~h}$. The beads were washed three times with the modified RIPA buffer. Samples were extracted by adding $4 \mathrm{X}$ of the sample buffer. Aliquots of the resulting samples were analyzed by SDS-PAGE and silver staining and then compared to identify proteins that were present in the collagen matrix samples but not in the trypsinized ones. Bands corresponding to the major affinity-selected proteins were identified by matrix-assisted laser desorption ionization-time of flight (MALDI-TOF) mass spectrometry.

$D D R 2$ silencing by siRNA. To knock down DDR2, primer pairs were designed by and obtained from Dharmacon (Chicago, IL, USA). siRNA silencing of gene expression in the cells was performed as previously described, with minor modifications (16). Mock-transfected cells were treated with only the sense direction oligonucleotide at a double concentration.

Immunofluorescence microscopy. Cell preparations for analysis were fixed for $10 \mathrm{~min}$ with $3 \%$ paraformaldehyde in phosphate-buffered saline (PBS), blocked with 2\% BSA/1\% glycine in PBS for $30 \mathrm{~min}$, and permeabilized for $15 \mathrm{~min}$ with $0.5 \%$ Triton X-100 in PBS. For actin staining, preparation of samples with Alexa Fluor 488-conjugated phalloidin and PI was carried out as previously described (17). Microscopic images were captured using a fluorescent microscope (Eclipse 80i; Nikon) using Plan Fluor 10X/0.30, Plan Apo 20X/0.75 and Plan Fluor 40X/0.75 infinity-corrected objectives. Images were acquired using a digital camera (digital sight DS-Qi1Mc; Nikon) and NIS element image analysis (Nikon). Image processing was carried out using Photoshop 11.0 (Adobe).

\section{Results and Discussion}

DDR2 is the major tyrosine-phosphorylated protein in $3 D$ collagen matrices. Based on the significance of phosphorylation on the tyrosine residues in a variety of signal proteins to transmit the intracellular signaling in response to specific environmental cues, we carried out the immunoprecipitation experiment with phospho-tyrosine antibodies using cell lysates that were prepared from 3D collagen matrices to identify the 3D environment-specific tyrosine-phosphorylated proteins and analyzed by MALDI-TOF mass spectrometry. Fig. 1A shows that several proteins increased the tyrosine phosphorylation in 3D collagen matrices. DDR2 $(\sim 134 \mathrm{kDa})$ was found to be the most prominent protein to increase the tyrosine phosphorylation in 3D collagen matrices. In particular, DDR2 enhanced the phosphorylation in response to PDGF stimulation. It has been reported that the DDRs only respond to extracellular 
A
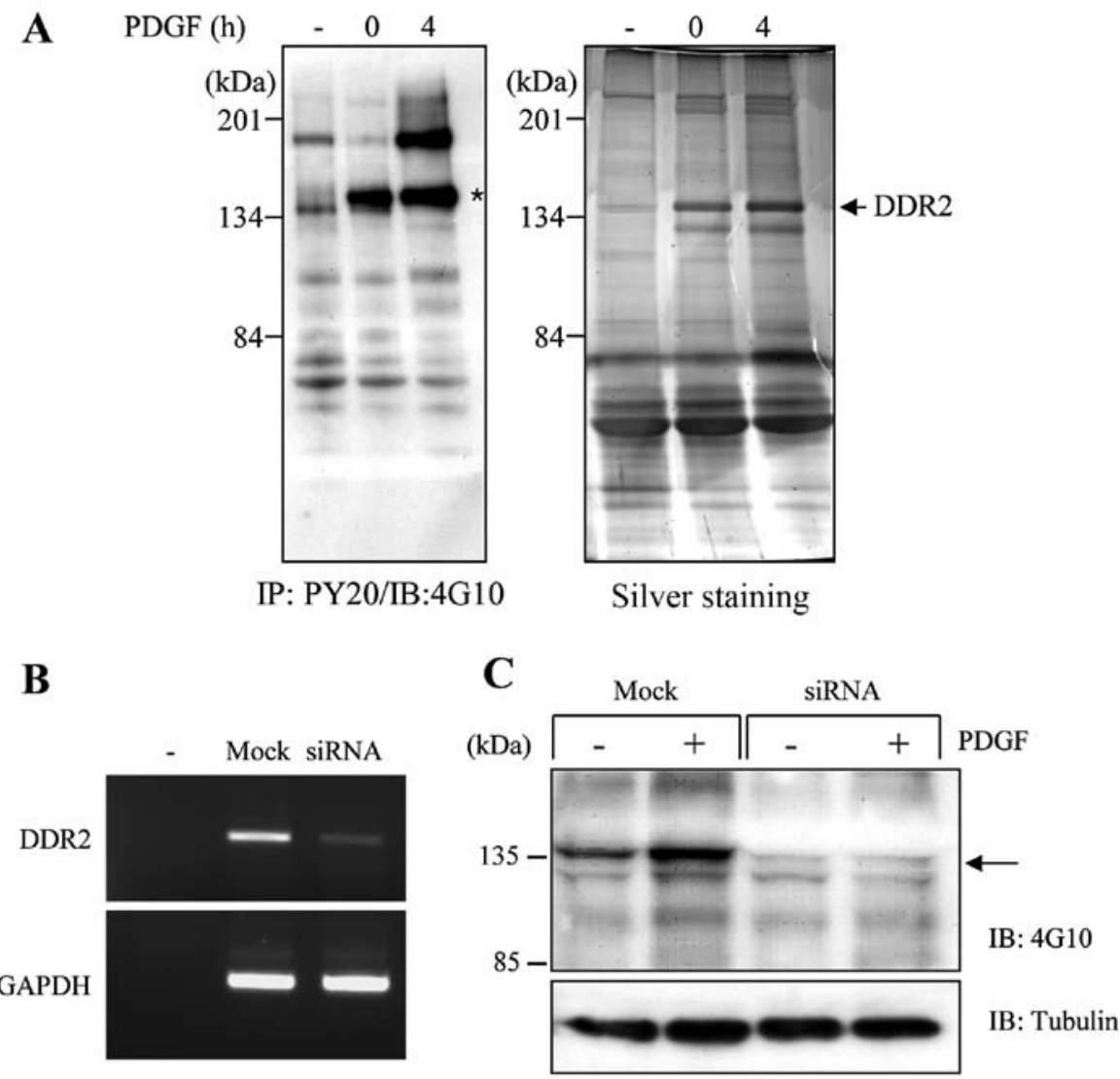

Figure 1. Phosphorylation of DDR2 in 3D collagen matrix. (A) Collagen matrices containing human fibroblasts were polymerized for $1 \mathrm{~h}$ (labeled $0 \mathrm{~h}$ ) and incubated with PDGF for $4 \mathrm{~h}$ as shown. At the end of the incubations, cells were harvested and immunoprecipitated using phosphotyrosine antibodies (PY20), after which half of the samples were subjected to immunoblotting with antibodies (4G10) to detect phosphotyrosine protein (left panel). The major tyrosinephosphorylated protein is indicated by an asterisk on the right. Half of the samples were subjected to silver staining and major precipitated proteins were analyzed by MALDI-TOF mass spectrometry (right panel). The results of the mass spectrometry are shown on the right. (B) RT-PCR showing that DDR2 siRNA, but not mock-transfected cells, specifically inhibited DDR2 expression. (C) Collagen matrices containing control and DDR2 silenced fibroblasts were incubated with PDGF for $4 \mathrm{~h}$. At the end of the incubations, lysates were prepared and subjected to immunoblotting with antibodies (4G10) directly against the phosphotyrosine protein. The arrow indicates that the phosphotyrosine protein of $135 \mathrm{kDa}$ size protein completely disappeared with DDR2 siRNA.

components such as fibrillar collagens, but not to soluble growth factors (12). However, our findings indicate that DDR2 appeared likely to increase the phosphorylation upon PDGF stimulation in 3D collagen matrices. Thus, it may be beneficial to define whether DDR2 can respond to soluble growth factors, such as PDGF, when cells are in the 3D environment.

To further analyze the role of DDR 2 in the 3D collagen matrices, we used siRNA technology to knock down DDR2 expression in the human fibroblasts. Fig. 1B provides an example of the RT-PCR analysis performed on the cell lysates prepared from the cells after $36 \mathrm{~h}$ of transfection with DDR2specific siRNA. The level of DDR 2 mRNA was markedly reduced, by almost $90 \%$, compared with that of the mocktransfected cells. We also confirmed the knockdown of DDR2 expression in the human fibroblasts using western blot analysis (data not shown). When DDR2 silencing cells were cultured in the $3 \mathrm{D}$ collagen matrices, the tyrosine-phosphorylated protein in a $135 \mathrm{kDa}$ size on the mock-transfected cells completely disappeared, indicating that DDR2 is the major tyrosinephosphorylated protein in $3 \mathrm{D}$ collagen matrices.

DDR2-silenced fibroblasts reduce expansion of dendritic extensions in $3 D$ collagen matrices. It has been shown that the PDGF causes the expansion of the dendritic network in fibroblasts, while LPA resulted in the retraction of dendritic extensions (16). Thus, we first examined the effect of DDR2 silencing on fibroblast morphology in 3D collagen matrices. Fig. 2A shows that the DDR2-silenced fibroblasts appeared to reduce the number of dendritic extensions, although the projected length of the dendritic extensions is similar to that of the control cells in response to PDGF stimulation. However, it had no effect on the LPA-mediated retraction of dendritic extensions in the fibroblasts. Fig. 2B shows the morphometric analysis of a representative experiment, which indicates that DDR2-silenced cells showed an $\sim 30 \%$ decrease in the number of dendritic extended cells compared with mock-transfected cells either in collagen or fibronectin matrices. These results suggest that DDR2 is involved in the regulation of fibroblast dendritic extensions but not in the formation of dendrites in the 3D collagen matrices.

We have shown that the microtubule-dependent mechanism is involved in cell spreading according to the tension state of the cell matrix interaction in 3D collagen matrices but actin dynamics are prerequisites for the initial spreading in this case (18). Our findings indicate that DDR2 silencing significantly inhibited dendritic extensions, indicating that it plays a critical role in determining the cell morphology in the $3 \mathrm{D}$ collagen matrices (Fig. 2A). Although there is a lack 
A

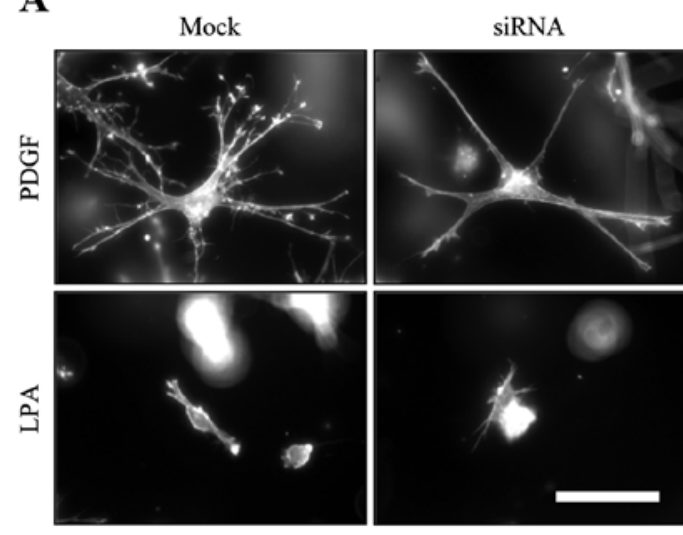

B

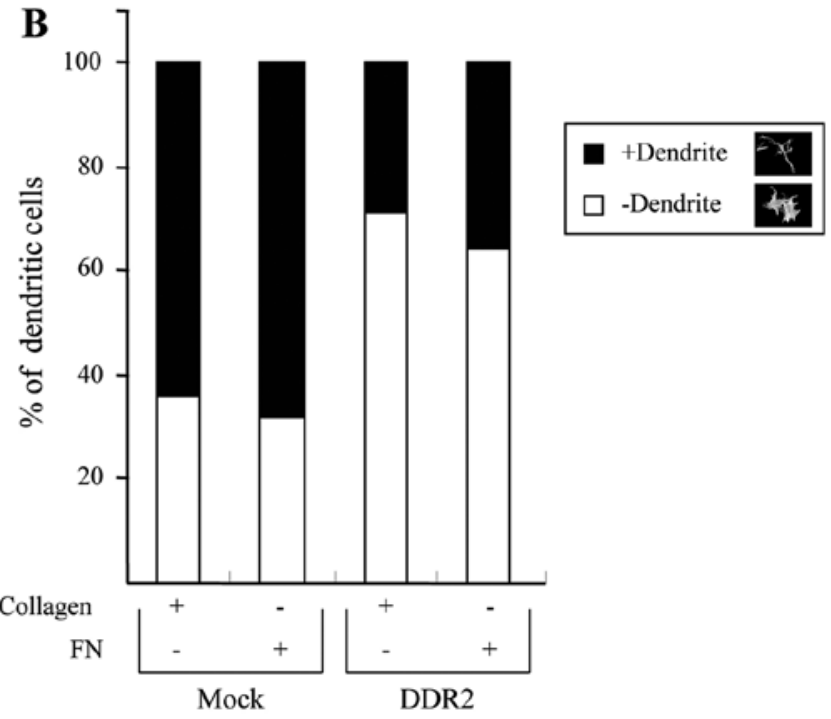

Figure 2. The effect of DDR2 silencing on dendritic extensions of fibroblasts. (A) Collagen or fibronectin (FN) matrices containing mock and DDR2 siRNAtransfected fibroblasts were polymerized for $1 \mathrm{~h}$ with $2 \times 10^{4}$ cells/matrix. Following polymerization, samples were incubated for $4 \mathrm{~h}$ in DMEM containing $50 \mathrm{ng} / \mathrm{ml}$ PDGF or $10 \mu \mathrm{M}$ LPA, as indicated. At the end of the incubations, samples were fixed and stained for actin. Selected images captured from collagen matrices are presented. Scale bar, $50 \mu \mathrm{m}$. (B) This is the same as in (A), except that samples were subjected to morphometric analysis. Cells were categorized as having $>5$ projected dendrites in the cell body as ' + ' dendrite as shown in the representative micrographs. A total of $30-50$ transfected cells were counted for each condition in $\sim 10$ fields. Results are given as percentages of cells in each category relative to the total number of cells counted.

of evidence regarding the relationship between DDR2 and cytoskeletal dynamics during cell spreading, it is possible that DDR may control the actin dynamics via myosin IIA in a dependent manner (15).

DDR2 silencing decreases the cell-matrix interaction in $3 D$ collagen matrices. Results indicated that the DDR2 silencing impaired the regulation of dendritic extensions of fibroblasts. Since it has been proposed that the attachment of fibroblasts to collagen fibrils in $3 \mathrm{D}$ collagen matrices is required for protruded dendrite (11), we examined the effect of DDR2 silencing on fibroblast attachment to $3 \mathrm{D}$ collagen matrices. Fig. 3A shows that the incubation with $10 \mathrm{mM}$ EDTA caused the removal of either mock or siRNA-transfected cells that had attached for 30 min to collagen-coated coverslips (11). However, fibroblasts that had attached to 3D collagen matrices for $30 \mathrm{~min}$ prior to EDTA treatment were unable to be released from the collagen matrices, whereas DDR2-silenced fibroblasts that were attached to the collagen matrices for $30 \mathrm{~min}$ were easily detached with the EDTA treatment (Fig. 3A), which suggests that the DDR2-mediated dendritic protrusion may be involved in the fibroblast and 3D collagen matrix interaction.

Fig. 3B presents representative images of mock and DDR2-silenced fibroblasts under control (PDGF) and EDTA conditions. After $1 \mathrm{~h}$ of incubation, the extensions of the dendrites in the DDR2-silenced cells were relatively simple and short in length compared with those of the control cells. However, a notable difference appeared in the EDTA-treated cells. The addition of EDTA to the control cells allowed the cells to form a small actin rich membrane protrusion and ruffles along the peripheral of plasma membrane, while DDR2 silencing resulted in complete inhibition of those morphological processes suggesting DDR2-mediated signaling is critical for calcium ion-independent cytoskeletal rearrangement and cell-matrix interaction.
It has been proposed that the cell adhesion to collagen matrices occurs in a divalent cation-independent manner (11). However, our results clearly showed that DDR2-silenced cell fibroblasts were completely removed from the 3D collagen matrices under the EDTA conditions. We also showed that the membrane protrusions in DDR2-silenced fibroblasts in the EDTA conditions were completely inhibited, although the mock-transfected cells exhibited tinny extensions of the membrane protrusions in the same conditions. Therefore, DDR2-mediated membrane protrusion may be involved in the integrin-independent cell adhesion in 3D collagen matrices.

DDR2 regulates matrix contraction and cell migration in $3 D$ collagen matrices. Lastly, we carried out an experiment to determine the ability of control and DDR2-silenced fibroblasts to contract the matrix. Fig. 4A shows the results of floating matrix contraction in response to various growth factors. Although it has been described in previous studies that the mechanical interaction of fibroblasts with collagen fibrils is critical for matrix contraction and cell migration in $3 \mathrm{D}$ collagen matrices (19), our results showed that the extent of growth factor dependence on matrix contraction with DDR2silenced cells is similar to that of mock-transfected cells, indicating that DDR2-dependent cell-matrix interaction may not to be required for the matrix contraction although there may be another mechanism to control.

To analyze the migration of DDR2-silenced and control fibroblasts in the 3D matrix environments, the pre-contracted collagen matrices shown in Fig. 4A were embedded in cellfree matrices to prepare the nested collagen matrices. The migration of cells in nested collagen matrices can be easily quantified when double stained for actin and PI by counting the number of nuclei moving out of the border of inner matrix (20). Fig. 4B shows representative images of the control and DDR2-silenced fibroblast migration after $24 \mathrm{~h}$ of migration 
A

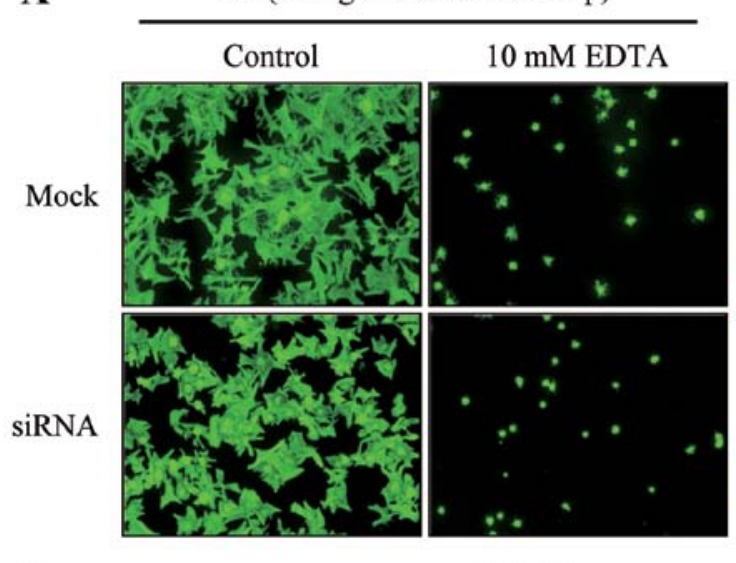

B

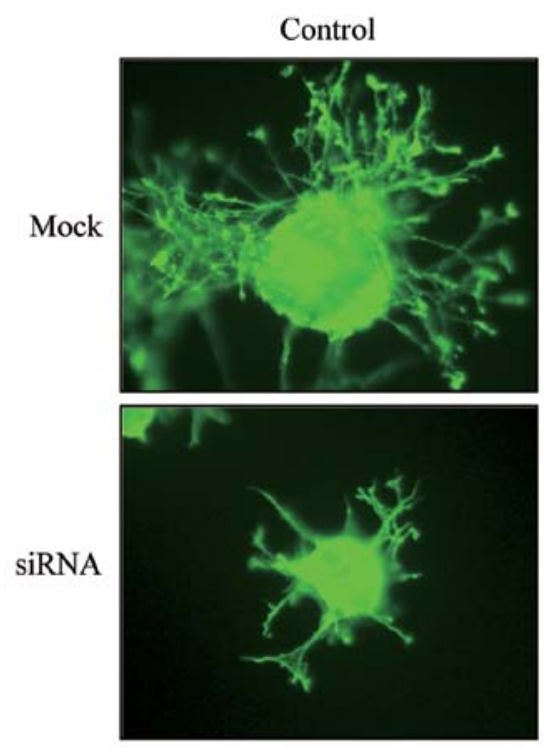

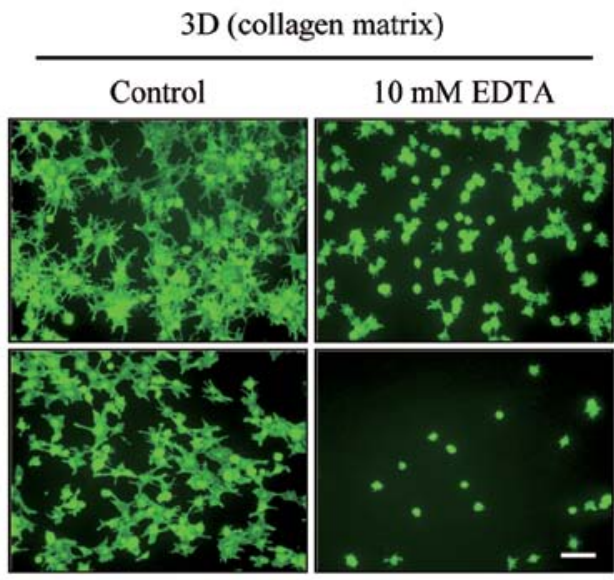

$10 \mathrm{mM}$ EDTA
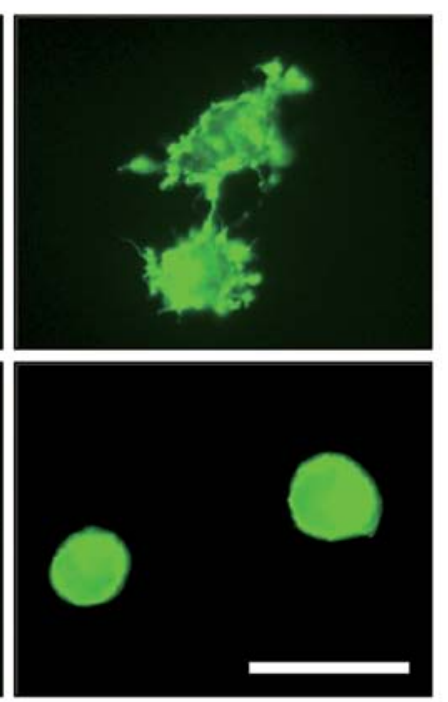

Figure 3. The effect of DDR2 silencing on the adhesion of fibroblasts to the 3D collagen matrix. (A) Mock and DDR2-silenced cells were attached for 30 min to collagen-coated coverslips or matrices, washed and then incubated with medium containing $10 \mathrm{mM}$ EDTA for $40 \mathrm{~min}$. At the end of the incubations, the samples were fixed and stained for actin. Scale bar, $100 \mu \mathrm{m}$. (B) An enlarged view of a single cell form elsewhere in (A). DDR2 silencing caused the inhibition of small protrusions induced by EDTA around the membrane, resulting in a weak interaction of fibroblasts to the 3D collagen matrices. Scale bar, $50 \mu \mathrm{m}$.

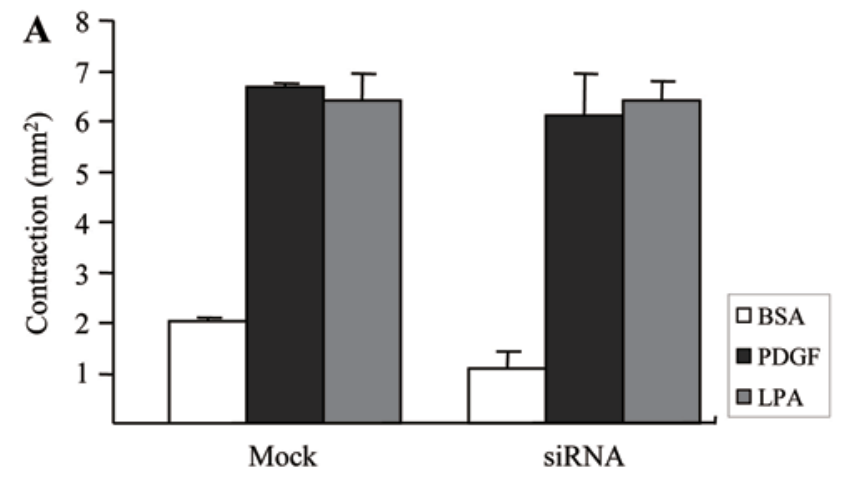

B

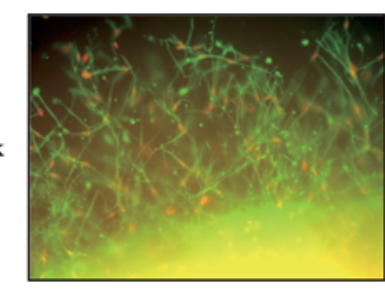

siRNA

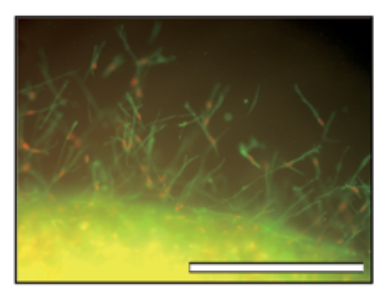

C

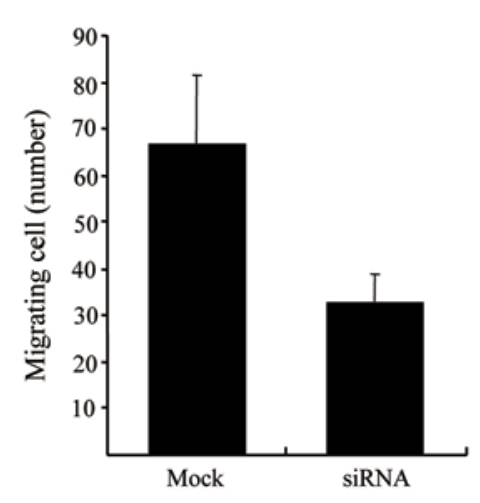

Figure 4. The effect of DDR2 silencing on fibroblast migration in a 3D environment. (A) Floating collagen matrices were prepared containing fibroblasts previously transfected (mock or DDR2 siRNA) as indicated. Samples were incubated for $6 \mathrm{~h}$ in DMEM with $5 \mathrm{mg} / \mathrm{ml}$ fatty acid-free BSA and $50 \mathrm{ng} / \mathrm{ml}$ PDGF or $10 \mu \mathrm{M} \mathrm{LPA}$ added as shown. At the end of the incubation period, samples were fixed and the extent of the matrix contraction was measured. Results shown are averages (means \pm SD) from three separate experiments, each carried out in duplicate. (B) Nested collagen matrices were prepared with precontracted floating matrices shown in (A), and incubated for $24 \mathrm{~h}$ in a medium containing PDGF. At the end of the incubation time, migrating cells were visualized by staining for actin (green) and propidium iodide (red). Scale bar, $100 \mu \mathrm{m}$. (C) Quantification of the cell migration shown in (B). Cell migration index values shown are the averages (means $\pm \mathrm{SD}$ ) of duplicate samples from three separate experiments. Compared to mock-transfected cells, DDR2-silenced fibroblasts showed decreased migration in the PDGF conditions. 
in PDGF-containing medium, and Fig. $4 \mathrm{C}$ presents the quantified results. DDR2-silenced fibroblasts markedly decreased cell migration in the 3D collagen environment, although they did not have a significant influence on the floating collagen matrix remodeling.

In the current study, we showed that DDR2 is required for the cell-collagen fibril interaction in 3D collagen matrices. It is unclear if the kinase activity or phosphorylation of DDR2 is necessary for DDR2 function in initial spreading and cell-matrix interaction in 3D collagen matrices, since the autophosphorylation appeared after 2-18 $\mathrm{h}$ of collagen stimulation (12). Previous studies reported that the tyrosine kinase-independent mechanism is sufficient to control the collagen fibrillogenesis and cell migration $(21,22)$, supporting the hypothesis that DDR2 for cell spreading and attachment in 3D collagen matrices may be mediated in a kinase-independent manner. It has been reported that the numerous signaling proteins, including PI3 kinase, Nck and Shc, are associated with the cytoplasmic region of DDR1 and form a large signaling complex either in a kinase-dependent or -independent manner but relatively little is known about the binding partners of DDR2 (14). Thus, it is possible that the signal proteins involved in cytoskeleton rearrangement could be recruited to the cytoplasmic region of DDR2 through which the signaling complex may play an important role in cell spreading and attachment in 3D collagen matrices. The fact that the DDR2-mediated signaling complex regulates fibroblast attachment to $3 \mathrm{D}$ collagen matrices is in agreement with a previous study which demonstrated that DDR1 regulated cell spreading and motility via myosin IIA (15). Moreover, DDR2 has an unusually long juxtamembrane domain in the cytoplasmic region, suggesting that it has an ability to serve as a dock site, making possible an intermolecular association. Thus, further studies to define the molecular interaction with the cytoplasmic domain of DDR2 are warranted.

\section{Acknowledgements}

We are particularly grateful to Dr Frederick Grinnell for the initiative effort toward this study. This study was supported by a 2010 Chung-Ang University research grant.

\section{References}

1. Engler AJ, Sweeney HL, Discher DE and Schwarzbauer JE: Extracellular matrix elasticity directs stem cell differentiation. J Musculoskelet Neuronal Interact 7: 335, 2007.

2. Friedl P and Bröcker EB: The biology of cell locomotion within three-dimensional extracellular matrix. Cell Mol Life Sci 57: 41-64, 2000.
3. Geiger B and Yamada KM: Molecular architecture and function of matrix adhesions. Cold Spring Harb Perspect Biol 3: a005033, 2011.

4. Levental KR, Yu H, Kass L, et al: Matrix crosslinking forces tumor progression by enhancing integrin signaling. Cell 139: 891-906, 2009.

5. Castelló-Cros R and Cukierman E: Stromagenesis during tumorigenesis: characterization of tumor-associated fibroblasts and stroma-derived 3D matrices. Methods Mol Biol 522: 275-305, 2009.

6. Hynes RO: The extracellular matrix: not just pretty fibrils Science 326: 1216-1219, 2009.

7. Larsen M, Artym VV, Green JA and Yamada KM: The matrix reorganized: extracellular matrix remodeling and integrin signaling. Curr Opin Cell Biol 18: 463-471, 2006.

8. Bershadsky AD, Ballestrem C, Carramusa L, et al: Assembly and mechanosensory function of focal adhesions: experiments and models. Eur J Cell Biol 85: 165-173, 2006.

9. Riveline, D, Zamir E, Balaban NQ, et al: Focal contacts as mechanosensors: externally applied local mechanical force induces growth of focal contacts by an mDia1-dependent and ROCKindependent mechanism. J Cell Biol 153: 1175-1186, 2001.

10. Grinnell F, Ho CH, Tamariz E, Lee DJ and Skuta G: Dendritic fibroblasts in three-dimensional collagen matrices. Mol Biol Cell 14: 384-395, 2003.

11. Jiang H and Grinnell F: Cell-matrix entanglement and mechanical anchorage of fibroblasts in three-dimensional collagen matrices. Mol Biol Cell 16: 5070-5076, 2005.

12. Vogel W, Gish GD, Alves F and Pawson T: The discoidin domain receptor tyrosine kinases are activated by collagen. Mol Cell 1: 13-23, 1997.

13. Vogel W: Discoidin domain receptors: structural relations and functional implications. FASEB J 13 (Suppl): S77-S82, 1999.

14. Vogel WF, Abdulhussein R and Ford CE: Sensing extracellular matrix: an update on discoidin domain receptor function. Cell Signal 18: 1108-1116, 2006.

15. Huang Y, Arora P, McCulloch CA and Vogel WF: The collagen receptor DDR1 regulates cell spreading and motility by associating with myosin IIA. J Cell Sci 122: 1637-1646, 2009.

16. Rhee S and Grinnell F: P21-activated kinase 1: convergence point in PDGF- and LPA-stimulated collagen matrix contraction by human fibroblasts. J Cell Biol 172: 423-432, 2006.

17. Kim D, You E and Rhee S: Dynein regulates cell migration depending on substrate rigidity. Int J Mol Med 29: 440-446, 2012.

18. Rhee S, Jiang $\mathrm{H}$, Ho $\mathrm{CH}$ and Grinnell F: Microtubule function in fibroblast spreading is modulated according to the tension state of cell-matrix interactions. Proc Natl Acad Sci USA 104: 5425-5430, 2007.

19. Provenzano PP, Inman DR, Eliceiri KW, Trier SM and Keely PJ: Contact guidance mediated three-dimensional cell migration is regulated by Rho/ROCK-dependent matrix reorganization. Biophys J 95: 5374-5384, 2008.

20. Grinnell F, Rocha LB, Iucu C, Rhee S and Jiang H: Nested collagen matrices: a new model to study migration of human fibroblast populations in three dimensions. Exp Cell Res 312: 86-94, 2006.

21. Blissett AR, Garbellini D, Calomeni EP, Mihai C, Elton TS and Agarwal G: Regulation of collagen fibrillogenesis by cell-surface expression of kinase dead DDR2. J Mol Biol 385: 902-911, 2009.

22. Hachehouche LN, Chetoui N and Aoudjit F: Implication of discoidin domain receptor 1 in $\mathrm{T}$ cell migration in three-dimensional collagen. Mol Immunol 47: 1866-1869, 2010. 